\title{
Semi-Empirical Equation to Describe the Electron Number Density in the Analytical Zone of the ICP as Functions of the r.f. Power, Height above the Load Coil, Carrier Gas and Water Flow Rates
}

\author{
Susumu NaKamURA \\ National Institute of Materials and Chemical Research, Higashi, Tsukuba 305-8565, Japan
}

\begin{abstract}
This study presents a semi-empirical equation of the electron number density at the analytical zone of the Ar ICP. This equation was developed from data of a three-stage experiment which demonstrated that the electron number density linearly increased with an increase in the r.f. power, exponentially decreased with an increase in the height above the load coil, and decreased when the quantity of the carrier gas and water molecules increased. This behavior of the electron number density at the analytical zone can be explained by the following equation: $n_{\mathrm{e}}=k_{\mathrm{p}} F\left[1-k_{5} \mathrm{~A}-k_{6} \mathrm{~W}\right] \exp \left(-k_{\mathrm{d}} x\right)$, where $A=A_{0}\left(1-k_{7} F\right) \exp \left(-k_{8} x\right)$ and $W=W_{0}\left(1-k_{9} F\right) \exp \left(-k_{10} x\right)$.
\end{abstract}

Keywords ICP atomic emission spectrometry, empirical equation, electron number density, analytical zone, dry or wet carrier gas

Many researchers have described mathematical models of the Ar ICP using fundamental parameters, such as the temperature, Ar flow rate and geometry of the ICP torch. ${ }^{1-10}$ Mathematical modeling of ICP is an excellent tool that has been used with considerable success in calculating the flow, temperature, and concentration fields in Ar ICP over a wide range of operating conditions. ${ }^{11}$ The use of mathematical models makes it possible to easily predict and describe the reaction behavior. If the Ar ICP is in a local thermal-equilibrium (LTE) condition, a mathematical model of the Ar ICP can be described by fundamental parameters of the Ar ICP. However, the Ar ICP deviates from the LTE conditions. A number of researchers have proposed mathematical models of the Ar ICP in the non-LTE condition, based on fundamental parameters. ${ }^{1,6,12}$ In the process of validating the mathematical models of the Ar ICP in the non-LTE condition, the temperatures predicted from the model were compared with the experimental data. The result indicated that the experimental data were in better agreement with a two-temperature model (electron and atoms/ions temperatures) than with the LTE model. Currently, the two-temperature model is regarded as being a valid mathematical model of the Ar ICP. ${ }^{1}$ However, the predicted temperatures from the two-temperature model do not explain experimental data perfectly. ${ }^{1}$ In order to eradicate any discrepancy between the experimental data and the data calculated by the mathematical model, it is necessary to use a fundamental approach, as well as an experimental approach. Yang and Barnes have recommended that the modeling of the behavior of particles as well as the temperatures in the Ar ICP is necessary for obtaining a correct mathematical modeling of the Ar ICP in the non-LTE condition. ${ }^{10}$

We are attempting to describe a mathematical model of the electron number density using empirical data. Our equation describes the electron number density of the analytical zone in the Ar ICP as a function of the r.f. power and the height ALC, as well as the quantity of carrier gas and water molecules. This equation was constructed in 3 stages of an experiment using an $\mathrm{Ar}$ ICP. In stage 1 , the electron number density was measured without carrier gas. In stage 2 , the electron number density was measured with a dry carrier gas (without water molecules). In stage 3 , the electron number density was measured with a wet carrier gas (with water molecules).

\section{Experimental}

The experimental conditions and procedure are almost the same as in a previous study. ${ }^{13}$ The equipment used in this study consisted of a $3 \mathrm{~kW}$ high frequency plasma generator (Model ICPS-2H, Shimadzu Co., Ltd., Kyoto, Japan) and a monochromator (Model CMT-100, Shimadzu Co., Ltd.) equipped with an R955 photomultiplier tube (Hamamatsu photonics Co., Ltd., Hamamatsu, Japan) as well as a 3600 grooves $\mathrm{mm}^{-1}$ diffraction grating. The entrance slit and the exit slit of the monochromator were set at a width of 10 and 20 $\mu \mathrm{m}$, respectively. The voltage of the photomultiplier tube was $500 \mathrm{~V}$. The ICP torch was $18 \mathrm{~mm}$ in diameter, and the coil diameter was $24 \mathrm{~mm}$ with two turns covering $10 \mathrm{~mm}$ in the axial direction. The flow rates of Ar 


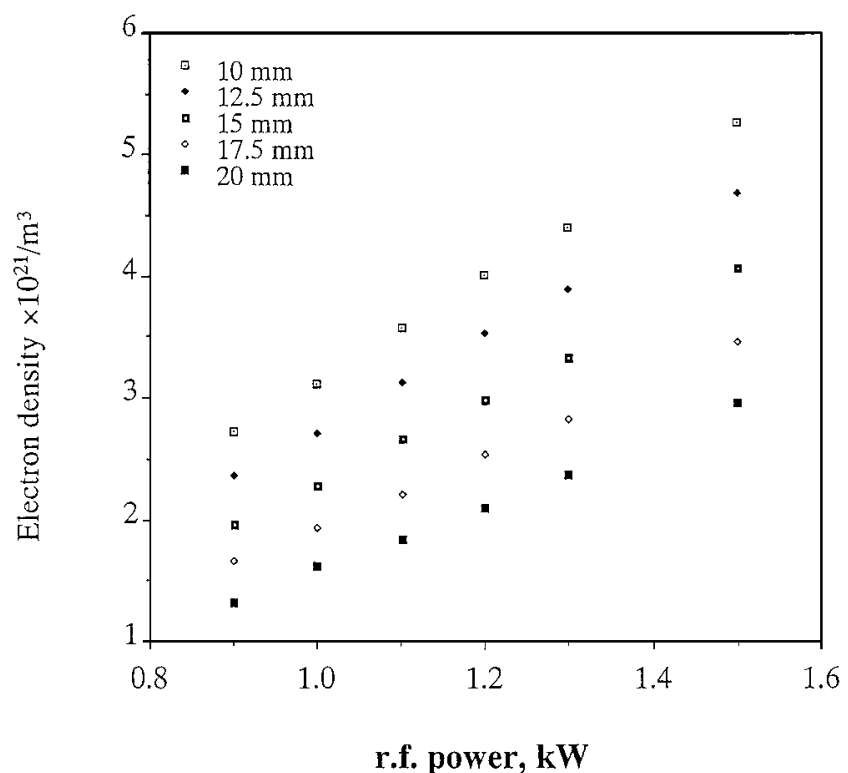

Fig. 1 Electron number density at the analytical zone without a carrier gas $v s$. r.f. power at five levels of the observation height above the load coil.

Table 1 Ar flow rates and water molecules in the carrier gas

\begin{tabular}{cccc}
\hline & $\begin{array}{c}\text { Experimental } \\
\text { stage }\end{array}$ & $\begin{array}{c}\text { Ar flow rate/ } \\
\text { mol min }^{-1}\end{array}$ & $\begin{array}{c}\text { Water molecules/ } \\
\text { mol min }\end{array}$ \\
\hline 1 & 1 & 0.0 & 0.0 \\
2 & 2 & 0.024 & 0.0 \\
3 & 2 & 0.033 & 0.0 \\
4 & 2 & 0.041 & 0.0 \\
5 & 3 & 0.024 & 0.049 \\
6 & 3 & 0.033 & 0.096 \\
7 & 3 & 0.041 & 0.121 \\
\hline
\end{tabular}

The quantites of water molecules are introduced into the nebulizer.

in the outer and intermediate chambers were 0.67 and $0.049 \mathrm{~mol} \mathrm{~min}^{-1}$, respectively. The experimental conditions, the flow rates of the carrier gas and the quantity of water molecules in the carrier gas are presented in Table 1. The electron number density was measured for six levels of the r.f. power $(0.9,1.0,1.1,1.2,1.3$ and $1.5 \mathrm{~kW})$, five levels of the observation height ALC (10, $12.5,15,17.5$ and $20 \mathrm{~mm}$ ), four flow rates of the carrier gas and three quantities of water. The electron number density distributions were inverted by the Abel equation. ${ }^{14-16}$

The electron number density was calculated by the continuum method (Eq.(1)) with the emission coefficient at $400 \mathrm{~nm}$ compared to the certified values of the standard deuterium lamp,

$$
\begin{aligned}
\mathcal{E}_{\mathrm{c}}(\lambda)=1.63 \times 10^{-34} & n_{\mathrm{e}}^{2} \lambda^{-2} T_{\mathrm{e}}^{-1 / 2} \xi \\
& \left(\mathrm{W} / \mathrm{m}^{3} \mathrm{~nm} \mathrm{~K}^{1 / 2} / \mathrm{sr}\right) .
\end{aligned}
$$

When using Eq. (1) to calculate the electron number density, $T_{\mathrm{e}}$ was substituted with $10000 \mathrm{~K}$ and $\xi$ was

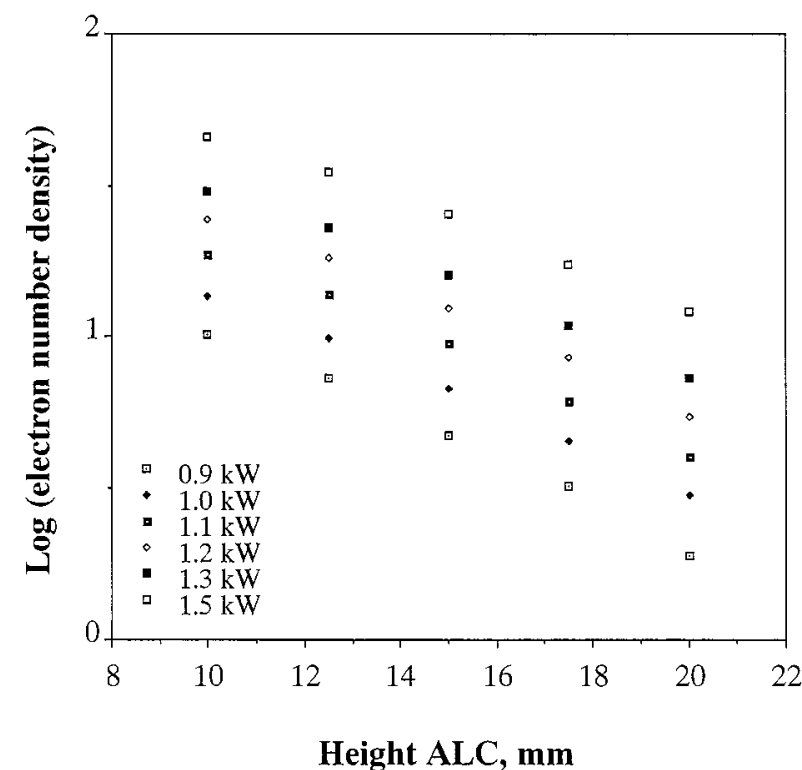

Fig. 2 Log(electron number density at the analytical zone without carrier gas) vs. observation height above the load coil at six levels of r.f. power.

substituted by $1.8 .^{17}$ The accuracy of the electron number density obtained by this method is on the order of $10 \% .^{17,18}$

\section{Results and Discussion}

Electron number density without a carrier gas as a function of the r.f. power and the observation height above the load coil

The electron number density of the analytical zone without a carrier gas was measured at six levels of the r.f. power and at five observation heights ALC. In this condition we did not observe the doughnut phenomena. The data of the current experiment indicate that the electron number density increases linearly with the r.f. power and that the logarithm of the electron number density decreases linearly with an increase in the observation height ALC (Figs. 1 and 2). These results are similar both in value and in tendency to the result obtained by using the Thomson scattering method ${ }^{19,20}$ and the Stark broadening method. ${ }^{21,22}$

The above-described behavior of electrons at the analytical zone can be explained by four assumptions: (1) electrons are created at the central area of the load coil by r.f. power; (2) a number of electrons react with ions and generate corresponding excited atoms and photons in a continuum of wavelengths; (3) the continuum emission intensity and r.f. power do not change with time; and (4) Ar flows without turbulence in the analytical zone.

Assumptions (1) - (4) imply that the electron number density is proportional to the linear increase in the r.f. power $(F)$. Assumptions (1) - (4) as well as the data of our experiment also lead to a differential equation of 


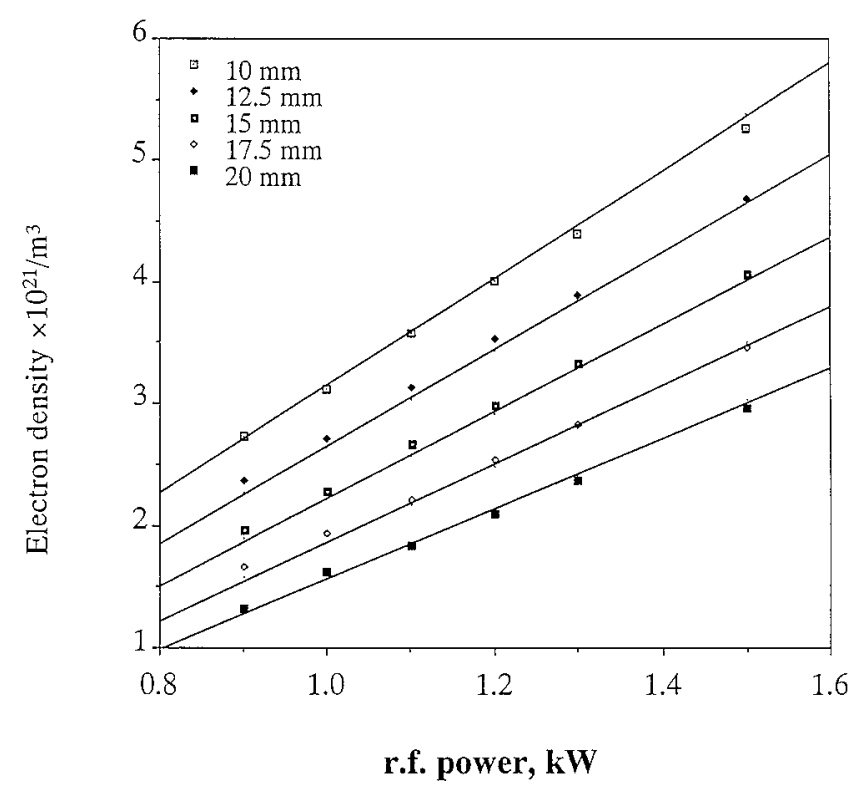

Fig. 3 Electron number density without a carrier gas vs. r.f. power at five levels of observation height above the load coil. The symbols represent the experimental data and the solid lines represent the values calculated by Eq. (3).

the electron number density in respect to the height $\left(\mathrm{d} n_{\mathrm{e}} / \mathrm{d} x\right)$, in which the electron number density is reduced as a function of the height ALC,

$$
n_{\mathrm{e}}=k_{\mathrm{p}} F \exp \left(-k_{\mathrm{d}} x\right),
$$

where $k_{\mathrm{p}}$ and $k_{\mathrm{d}}$ are constant factors.

If Eq. (2) is a valid explanation of the electron number density without a carrier gas at the analytical zone in the Ar ICP, the constant factors, $k_{\mathrm{p}}$ and $k_{\mathrm{d}}$, should be unit numbers under various conditions of the r.f. power and height ALC. Using least-squares methods and Eq. (2), we demonstrated that in our experiment $k_{\mathrm{d}}$ and $k_{\mathrm{p}}$ have constant values $\left[k_{\mathrm{p}}=(6.35 \pm 1.4) \times 10^{21}\right.$ and $\left.k_{\mathrm{d}}=0.07(F)^{-1 / 2}\right]$. Therefore, the electron number density in our experiment without a carrier gas is represented by

$$
n_{\text {e-without }}=6.35 \times 10^{21} F \exp \left[-0.07(F)^{-1 / 2} x\right] .
$$

Figure 3 shows the experimental data and values calculated by Eq. (3).

\section{Electron number density as a function of the quantity of dry and wet carrier gases}

The electron number density decreases with an increase in the quantity of carrier gas and water molecules. This tendency is similar to the condition of the electron number density without a carrier gas (Fig. 4). This behavior can be explained by assumptions (1) - (4) above and by one additional assumption: electrons are quenched by the carrier gas and water molecules. These five assumptions lead to a equation of the electron number density with a carrier gas and water molecules at the analytical zone:

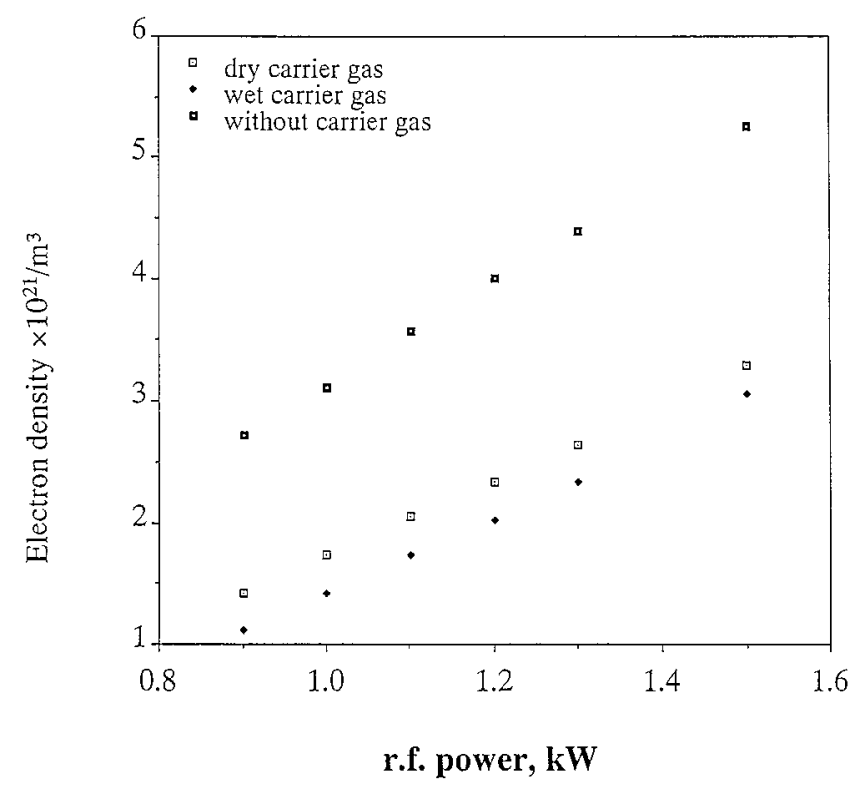

Fig. 4 Electron number density without a carrier gas (without) and with a $0.041 \mathrm{~mol} \mathrm{~min}^{-1}$ carrier gas with water (wet), or without water (dry) plotted against r.f. power at $10 \mathrm{~mm}$ height above the load coil.

$$
\begin{gathered}
\mathrm{n}_{\mathrm{e}}=k_{\mathrm{p}} F\left[1-k_{5} A-k_{6} W\right] \exp \left(-k_{\mathrm{d}} x\right), \\
A=A_{0}\left(1-k_{7} F\right) \exp \left(-k_{8} x\right), \\
W=W_{0}\left(1-k_{9} F\right) \exp \left(-k_{10} x\right),
\end{gathered}
$$

where $k_{5}, k_{6}, k_{7}, k_{8}, k_{9}$ and $k_{10}$ are constant factors. A and $W$ represent the quantity of carrier gas and water molecules at each analytical point of the plasma. $A_{0}$ and $W_{0}$ represent the quantity of carrier gas and water molecules introduced into the nebulizer.

If Eq. (4) is a valid explanation of the electron number density at the analytical zone in the Ar ICP, the constant numbers, from $k_{5}$ to $k_{10}$, should have values that are invariable under various conditions of the r.f. power, height ALC, quantity of carrier gas and water molecules.

Dry carrier gas condition. According to Eq. (4), the electron number density with a dry carrier gas $\left(W_{0}=0\right)$ is obtained by the following equation:

$$
\begin{aligned}
& n_{\mathrm{e}}=k_{\mathrm{p}} F\left[1-k_{5} A\right] \exp \left(-k_{\mathrm{d}} x\right), \\
& A=A_{0}\left(1-k_{7} F\right) \exp \left(-k_{8} x\right) .
\end{aligned}
$$

To obtain $k_{8}$, the electron number density with a dry carrier gas was compared to the electron number density without a carrier gas:

$$
\begin{aligned}
& n_{\mathrm{e} \text {-dry }} / n_{\mathrm{e} \text {-without }}=1-k_{5} A_{0}\left(1-k_{7} F\right) \exp \left(-k_{8} x\right), \\
& \log \left[1-\left(n_{\mathrm{e} \text {-dry }} / n_{\mathrm{e} \text {-without }}\right)\right]=-k_{8} x+\log \left[k_{5} A_{0}\left(1-k_{7} F\right)\right],
\end{aligned}
$$

where $n_{\text {e-dry }}$ and $n_{\text {e-without }}$ are the electron number density 
with a dry carrier gas and without a carrier gas, respectively. According to the experimental result, there was only a small change $(<0.02)$ in $\log \left[1-\left(n_{\mathrm{e}-\text { dry }} / n_{\mathrm{e} \text {-without }}\right)\right]$ as a function of the height ALC; therefore, $k_{8}$ was close to zero. Using least-squares methods and

$$
1-\left(n_{\text {e-dry }} / n_{\text {e-without }}\right)=k_{5} A_{0}\left(1-k_{7} F\right)
$$

we could obtain $k_{5}$ and $k_{7}$ are $14.6 \pm 3.0$ and $0.25 \pm 0.05$, respectively.

Wet carrier gas condition. The electron number density also decreases along with an increase in the quantity of water molecules. To obtain $k_{6}, k_{9}$ and $k_{10}$, the electron number density with a wet carrier gas was compared to the electron number density with a dry carrier gas:

$$
\begin{aligned}
n_{\text {e-dry }} / n_{\text {e-without }}= & 1-k_{5} A_{0}\left(1-k_{7} F\right) \exp \left(-k_{8} x\right), \\
n_{\text {e-wet }} / n_{\text {e-without }}= & 1-k_{5} A_{0}\left(1-k_{7} F\right) \exp \left(-k_{8} x\right) \\
& -k_{6} W_{0}\left(1-k_{9} F\right) \exp \left(-k_{10} x\right),
\end{aligned}
$$

where $n_{\text {e-dry }}, n_{\text {e-wet }}$ and $n_{\text {e-without }}$ are the electron number density with a dry carrier gas, a wet carrier gas and without a carrier gas, respectively. The effect of water molecules is obtained by substracting Eq. (B) from Eq. (A),

$$
\begin{aligned}
\left(n_{\text {e-dry }} / n_{\text {e-without }}\right)-( & \left(n_{\text {e-wet }} / n_{\text {e-without }}\right) \\
= & k_{6} W_{0}\left(1-k_{9} F\right) \exp \left(-k_{10} x\right) .
\end{aligned}
$$

We measured the electron number density under two conditions: 1) with a dry carrier gas and 2) with a wet carrier gas. The data from these two conditions were nearly identical to each other. According to the experimental result, there was only a small change $(<0.03)$ in $\log \left[\left(n_{\text {e-dry }} / n_{\text {e-without }}\right)-\left(n_{\text {e-wet }} / n_{\text {e-without }}\right)\right]$ as a function of the height ALC; therefore, $k_{10}$ was close to zero. Using least-squares methods and

$$
\left(n_{\text {e-dry }} / n_{\text {e-without }}\right)-\left(n_{\text {e-wet }} / n_{\text {e-without }}\right)=k_{6} A_{0}\left(1-k_{9} F\right)
$$

we could obtain $k_{6}$ and $k_{9}$ are $1.7 \pm 0.3$ and $0.5 \pm 0.1$, respectively.

According to this result, the effect of the electron number density with water molecules in the carrier gas was less than $10 \%$ compared to the effect of the Ar carrier gas. Hence, the electron number density, including carrier gas with water, is represented by

$$
\begin{aligned}
& n_{\mathrm{e}}=6.35 \times 10^{21} F\left[1-14.6 A_{0}(1-0.25 F)-\right. \\
& \left.1.7 W_{0}(1-0.5 F)\right] \exp \left[-0.07(F)^{-1 / 2} x\right] .
\end{aligned}
$$

Figure 5 shows that the experimental data and the values calculated by Eq. (7).

This study presents a semi-empirical equation of the electron number density at the analytical zone. This specific equation (Eq. (7)) can be used to calculate the electron number density using a plasma torch with a

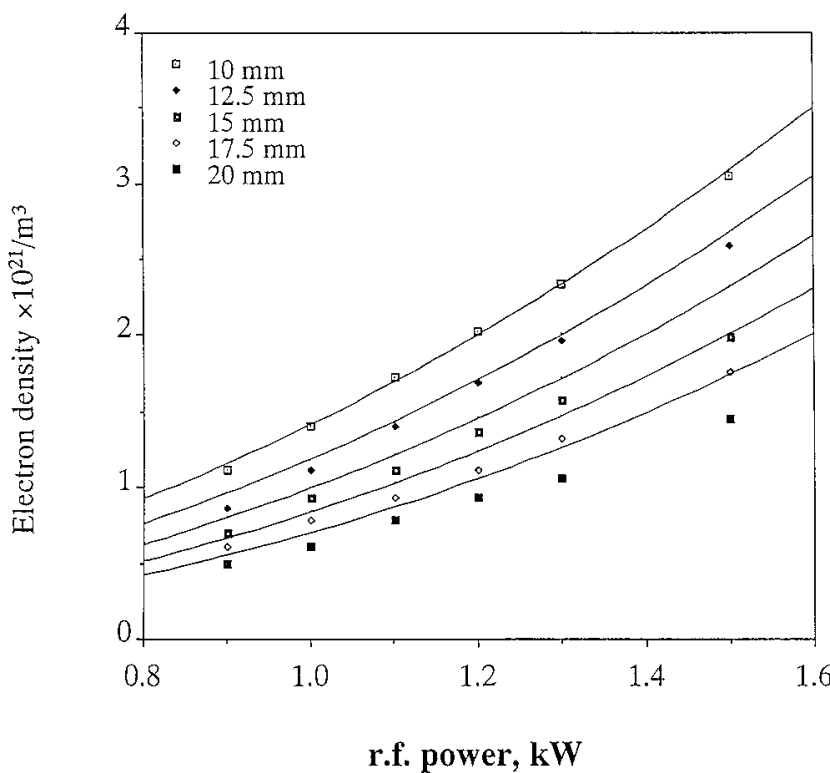

Fig. 5 Electron number density with $0.041 \mathrm{~mol} \mathrm{~min}^{-1}$ carrier gas $v s$. r.f. power at five levels of the observation height above the load coil. The symbols represent the experimental data and the solid lines represent the values calculated by Eq. (6).

diameter of $18 \mathrm{~mm}$ as functions of the r.f. power, the observation height ALC, and the quantity of the carrier gas and water molecules. It is predicted by this equation that the electron number density at the analytical zone increases linearly with an increase in the r.f. power, and exponentially decreases with an increase in the height ALC. In addition, it is predicted that the electron number density decreases when the quantity of carrier gas and water molecules increases. Unfortunately, the electron number density using plasma torches with other diameters, such as $13 \mathrm{~mm}$, cannot be calculated using Eq. (7). However, Eq. (4) can represent the electron number density when using a plasma torch with a $13 \mathrm{~mm}$ diameter as functions of the r.f. power, the observation height ALC, and the quantity of carrier gas and water molecules.

The precision of the numerals in Eq. (7) was approximately $20 \%$. This variance can be caused by the variance in the experimental data.

The equation in this study represents the conditions of the Ar ICP without analytes and matrixes. Our final goal is to develop an equation which can represent the conditions all over the Ar ICP with analytes and matrixes included. However, such an equation is very complex. This is because when analytes and matrixes are in the Ar ICP, the electron number density may differ from the electron number density when they are absent. If it is possible to express the difference of the electron number density by an equation when the analytes and matrixes are present, the excitation and quenching mechanisms in the Ar ICP can also be explained by using the same equation. Furthermore, if the equation can be used to explain the behavior of the analytes, the reaction mechanisms in the Ar ICP will 
become clearer. In our laboratory, we have initiated an experiment to expand the equations described in this paper to represent the conditions in the Ar ICP when analytes and matrixes are included.

\section{References}

1. J. Mostaghimi and M. I. Boulos, J. Appl. Phys., 68, 2643 (1990).

2. M. I. Boulos and R. M. Barnes, in "Inductively Coupled Plasma Emission Spectrometry", Part II, ed. P. W. J. M. Boumans, p. 289, Wiley, New York, 1987.

3. X. Chen, Int. J. Heat Transfer, 33, 815 (1990).

4. P. Yang, R. M. Barnes, J. Mostaghimi and M. I. Boulos, Spectrochim. Acta, 44B, 657 (1989).

5. M. El-Hage, J. Mostaghimi and M. I. Boulos, J. Appl. Phys., 65, 4178 (1989).

6. J. Mostaghimi P. Proulx and M. I. Boulos, J. Appl. Phys., 61, 1753 (1987).

7. I. Izumi, H. Tan, S. Chan and A. Montaser, Spectrochim. Acta, 46B, 901 (1991).

8. G. Y. Zhao, J. Mostaghimi and M. I. Boulos, Plasma Chem. Plasma Process, 10, 133 (1989).

9. G. Y. Zhao, J. Mostaghimi and M. I. Boulos, Plasma Chem. Plasma Process, 10, 151 (1989).

10. P. Yang and R. M. Barnes, Spectrochim. Acta Rev., 13, 27
(1990).

11. J. Mostaghimi and M. I. Boulod, in "Inductively Coupled Plasmas in Analytical Atomic Spectrometry", 2nd ed., ed. A. Montaser and D. W. Golightly, p. 949, VCH, Publishers, Inc., Cambridge, UK, 1992.

12. S. H. Paik and E. Pfender, Plasma Chem. Plasma Process., 10, 167 (1990)

13. S. Nakamura, Anal. Sci., 12, 707 (1996)

14. G. R. Kornblum and L. de Galan, Spectrochim. Acta, 29B, 249 (1974).

15. J. Jarosz, J. M. Mermet and P. J. Robin, Spectrochim. Acta 33B, 55 (1978)

16. K. Bockasten, J. Opt. Soc. Am., 51, 943 (1961).

17. G. J. Bastiaans and R. A. Mangold, Spectrochim. Acta, 40B, 885 (1985).

18. H. R. Griem, "Plasma Spectroscopy", McGraw-Hill, New York, 1964.

19. J. W. Olesik and S.-J. Den, Spectrochim. Acta, 45B, 731 (1990).

20. M. Huang and G. M. Hieftje, Spectrochim. Acta, 44B, 291 (1989).

21. B. Budic and V. Hudnik, J. Anal. At. Spectrom., 9, 53 (1994).

22. S. E. Long and R. F. Browner, Spectrochim. Acta, 43B, $1461(1988)$

(Received February 18, 1999) (Accepted April 28, 1999) 